\title{
3D model of flow in micro channels
}

\author{
Tatiana Kudryashova* and Sergey Polyakov \\ Keldysh Institute of Applied Mathematics RAS, Miusskaya sq. 4, Moscow 125047, Russia
}

\begin{abstract}
The micro channels are important components in many micro - electro - mechanical systems (MEMS) and technical systems. They are used to transport a gas or a liquid into devices and the systems. In our work, the gas flows into a micro channel have been studied for various shapes and sizes of the micro channels. Numerical simulations of compressible flows through a micro channel are performed by solving the Navier-Stokes equations and the quasigasdynamic equations (QGD). The numerical approaches are realized on hybrid parallel computer systems. The software includes the Domain Decomposition technique, the Message Passing Interface (MPI) for organization of interprocess data exchange and an application programming interface Open Multi-Processing (OpenMP). The calculations are performed on the Heterogeneous Computer Systems with Video Processing Units (VPU) and the classical microprocessors Central Processing Unit (CPU).
\end{abstract}

\section{Introduction}

Earth's limited resources make resource saving and energy saving not only a priority for the development of civilization, but also the guarantee of its survival. One of the main directions of realization of this priority is the creation of micro and nanotechnologies with enhanced performance characteristics. The advantages of these technologies are diminutiveness, low power consumption, low material consumption, reliability, demand across all sectors of the economy.

These technologies can be applied in medicine, for creation of new materials, for computer techniques of a new generation, for purification of water and air and etc.

MEMS technologies have typical sizes from 1 micrometer $(\mu \mathrm{m})$ up to a few millimetres. The complexity of such systems depends on the number of components. Nowadays, MEMS can include about several millions of units. Today, there are nano - electro - mechanical systems (NEMS). These systems have submicron sizes. MEMS and NEMS have the same functional part, but NEMS work with spatial (or time) scales about 2-3 orders less.

MEMS and NEMS technologies are applied to create micro sensors, engines, generators, pumps, filters and etc. These complicated systems are needed to study in details. For this analysis, we should join the experiment results and mathematical modelling. In contrast to many other computational challenges, applications in micro and nano technologies typically have specific problem size [1].

This paper deals with approaches to calculate gas flows in the micro channels of the technical systems. We study the flows for various configurations of the micro channels. The calculations were performed for laminar flow in the micro channels with the cross sections: circular, rectangular and annular channel [2].

There are many studies about circular micro channels although the rectangular micro channels have more prospects for being used in the technical systems. They are easier to produce, more technological and more simple for mathematical modelling.

The goal of this study is investigation of characteristics of flow for various configurations of the micro channels and comparison of two numerical approaches. For computing we used the QGD equations and the Navier-Stokes equations.

The numerical results will be compared with the flow regimes into the micro channels of the various sizes and shapes of the cross-section.

In technical applications the problems with the large Knudsen numbers and the high Reynolds numbers have appeared. These tasks have a complex real geometry. To solve these problems, fine unstructured (irregular) grids and hybrid grids are used. Such computational challenges require the utilization of modern hybrid parallel computer systems. In our work, two computing systems are implemented with VPU and CPU microprocessors.

\section{Governing equations}

The numerical interpretation is based on the system of the QGD equations [3, 4]. The QGD equations are obtained by a kinetic approach. The Boltzman equation is integrated with collision invariants, using a special variant of serial expansion for the distribution function. The system of QGD equations has been constructed as an extension of the traditional Navier-Stokes system of equations. This model generalizes the Navier-Stokes

* Corresponding author: kudryashova@imamod.ru

C The Authors, published by EDP Sciences. This is an open access article distributed under the terms of the Creative Commons Attribution License 4.0 (http://creativecommons.org/licenses/by/4.0/). 
system and it is different from the Navier-Stokes system in additional dissipative terms with a small parameter $\tau$. The system of the QGD equations is used for the flow field calculations [5]. Efficiency and precision of this method are provided by validity of conservation laws and entropy balance. With the usual notations, the general form of the QGD system is written as:

$$
\begin{aligned}
& \frac{\partial \rho}{\partial t}+\operatorname{div} \mathbf{j}=0, \quad \mathbf{j}=\rho(\mathbf{u}-\mathbf{w}), \\
& \mathbf{w}=\tau\left[\frac{1}{\rho} \operatorname{div}(\rho \mathbf{u})\right] \mathbf{u}+\mathbf{w}^{*}, \\
& \mathbf{w}^{*}=\tau\left[(\mathbf{u} \nabla) \mathbf{u} \frac{1}{\stackrel{+}{\rho}} p\right], \nabla
\end{aligned}
$$

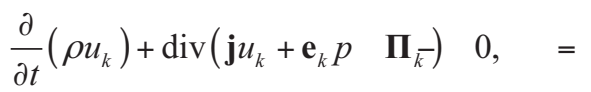

$$
\begin{aligned}
& \Pi_{k}=\left(\Pi_{j, k}\right), \quad \Pi_{k, \bar{j}}=\Pi_{k, j}^{N S} \Pi_{k, j}^{A D}, \\
& \Pi_{k, j}^{N S}=\mu\left(\frac{\partial u_{k}}{\partial x_{j}}+\frac{\partial u_{j}}{\partial x_{k}}+\delta_{k, j}\left(\zeta \quad \frac{2}{3}\right) \operatorname{div}(\mathbf{u})\right), \\
& \Pi_{k, j}^{A D}=\rho u_{k} w_{j}^{*}+\delta_{k, j} \nabla((\mathbf{u}) p), \\
& j, k=1,2,3 \text {; } \\
& \frac{\partial}{\partial t} E+\operatorname{div}(\mathbf{j} H+\mathbf{q} \quad \Pi \mathbf{\Pi u})-0, \quad= \\
& \mathbf{q}=-\kappa \nabla T-\tau\left[(\mathbf{u} \nabla)+\varepsilon \nabla p(\mathbf{u})\left(\frac{1}{\rho}\right)\right] \rho \mathbf{u}, \\
& E=\rho\left(\frac{1}{2}|\mathbf{u}|^{2}+\varepsilon\right), \quad \varepsilon=c_{V} T, \\
& H=\frac{E+p}{\rho}, \quad p=Z \rho \quad T, \quad \Re \\
& \gamma=\frac{c_{p}}{c_{V}}, \quad \tau=\frac{\mu}{p \mathrm{Sc}} \\
& \operatorname{Pr}=\frac{\mu c_{p}}{\kappa}, \quad \mathrm{Sc}=\frac{\mu}{\rho D} .
\end{aligned}
$$

Here $\rho$ is the density, $\mathbf{u}$ is the velocity vector, $p=\rho R T$ the pressure, $\Re$ is the universal gas constant, $\mathrm{T}$ is the temperature, $\varepsilon$ is the internal energy, $\gamma$ is the specific heat ratio, $\mathrm{H}$ is the enthalpy, $\mu, \zeta, \kappa, \tau$ are the kinetic coefficients of gas $(\mu$ is the coefficient of dynamic viscosity, $\zeta$ is the coefficient of relative volume viscosity, $\kappa$ is the coefficient of thermal conductivity of gas, $\tau$ is the relaxation time of the flow to a quasiequilibrium state). Dependencies of these coefficients on the governing parameters are written as follows: $\mu=\mu(T), \quad \xi=\zeta(T), \quad \chi=\chi(T)$ and $\tau=\tau(T, \rho)$. $Z=Z(T, \rho)$ is compressibility factor, $\gamma=\gamma(T, \rho)$ is adiabatic index, $c_{V}=c_{V}(\not), c_{p}=c_{p}(\hbar)$ are specific heat capacity and $\Re=k_{B} / m$ is the gas constant, $\left(k_{B}\right.$ is the Boltzmann's constant, Sc and $\mathrm{Pr}$ are the Schmidt and the Prandtl numbers); $\mathbf{j}$ and $\boldsymbol{\Pi}$ are modified fluxs of density and viscous stress tensor; $\rho \mathbf{w}$ and $\rho \mathbf{w}^{*}$ are vectors of the QGD corrections $\rho \mathbf{u} ; \mathbf{q}$ is heat flux vector; $\mathbf{e}_{k}(k=1,2,3)$ are the orthogonal unit vectors, $\operatorname{div}$ and $\nabla$ are the operators of divergence and gradient. To analyze the results of calculations, we need the values of flow parameters such as the Mach number, the Reynolds number and the Knudsen number.

$$
\begin{aligned}
& a^{2}=\gamma \frac{p}{\rho}, \quad \lambda \approx \frac{\mu}{\rho \sqrt{Z \Re T}} A, \\
& \mathrm{Ma}=\frac{|\mathbf{u}|}{a}, \quad \operatorname{Re}=\frac{\rho|\mathbf{u}| \lambda}{\mu}, \quad K n=\frac{L}{\lambda} .
\end{aligned}
$$

Where $a-$ the speed of sound and $\lambda$ - the mean free path. The system (1) - (3) can be completed by initial and boundary conditions. The initial conditions correspond to an equilibrium state of the gas medium without interaction with external factors.

The initial conditions are :

$$
\rho=\rho_{0}, \quad \mathbf{u}=0, \quad P=P_{0}, \quad T=T_{0} .
$$

Here $\rho_{0}$ and $p_{0}$ - initial gas density and pressure, $T_{0}$ is the initial temperature. Inlet parameters are:

$$
(\mathbf{u}, \mathbf{n})=u_{i n}, \quad \rho=\rho_{i n}, \quad T=T_{i n} .
$$

On free surfaces of the computational domain, the socalled "soft" boundary conditions can be written as:

$$
\frac{\partial \rho}{\partial n}=0, \quad \frac{\partial(\rho \mathbf{u})}{\partial n}=0, \quad \frac{\partial p}{\partial n}=0 .
$$

For solid walls sliding conditions were applied.

If $\tau$ is equal to 0 , we have the Navier-Stokes system of equations.

\section{Problem description}

The problem is considered for calculation of the nitrogen flow across the nozzle in a micro channel. The experimental data for the issue was published in [6]. The computational domain is shown in Figure. 1.

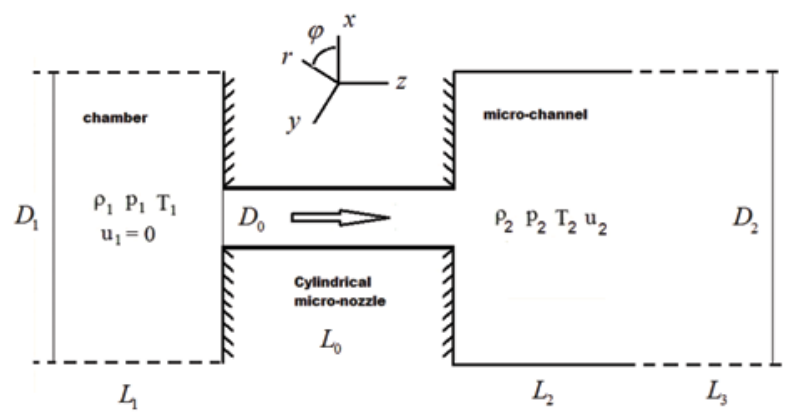

Fig. 1. Computational domain. 
The cylindrical micro nozzle has the diameter $D_{0}$ and length $L_{0}=6 D_{0}$. The computational domain has the diameter $D_{1}=6 D_{0}$ and lengths $L_{1}=10 L_{0}, L_{2}=30 L_{0}$ and $L_{3}=20 L_{0}$. It connects the chamber with nitrogen and micro-channel. At an initial moment $\mathrm{t}=0$ the gas is at rest: $u_{1}=u_{2}=0$. In the chamber nitrogen is located under standard normal conditions: $T_{1}=295.15 \mathrm{~K}$, $p_{1}=101325 \mathrm{~Pa}$. The gas is disposed in the nozzle and chamber with temperature $T_{2}=295.15 \mathrm{~K}$ and the pressure, $p_{2}=\delta_{0} p_{1}, \delta_{0} \quad 10^{-3} \div 10^{-5}$. The nozzle on the left is blocked by a partition, which opens instantly at the beginning of the calculation. The inner surface of the nozzle is perfectly smooth and thermally insulated. The diameters of the computational areas of the cylinder micro nozzle and the vacuum chamber were equal: $D_{1}=D_{2}=6 D_{0}$. The calculated part length of the cylinder micro nozzle is $L_{1}=10 L_{0}$; the calculated area length of the vacuum chamber is $L_{2}=50 L_{0}$.

For computing, various sizes were implemented. In particular, the value of diameter $D_{0}$ was varied from 3.1 - 310 micrometers. The results of the numerical experiments are presented in the sections below.

\section{Numerical method}

The grid numerical method has a finite volume method as basis and is used to calculate the macro parameters on the QGD equations [7-9]. For this purpose, in the computational domain $D$, the spatial grid $\Omega_{D}$ with cells $C_{m}(m=1, \ldots, M)$ and time grid $\omega_{t}$ with variable step $\Delta t$ was introduced. The grid $\Omega_{D}$ is a hybrid mesh, that is, it includes several element types.

All parameters of the gas components (density, pressure, temperature, velocity vector components, etc.) have been carried to the centers of mass of grid elements, that is, to the centers of cells. Stream variables have been set in the centers of the cells sides. Spatial approximations of the main terms have been executed by the technique presented in work [10]. The computing scheme on time is obvious. The resulting grid equations at a predictor stage have view:

$$
\begin{aligned}
& \hat{\rho}_{l, m}=\rho_{l, m}+\frac{\Delta t}{\left|V_{m}\right|} \sum_{p=1}^{M_{m}} W_{l, n, m, p}^{(\rho)}\left|S_{m, p}\right|, \\
& W_{l, n, m, p}^{(\rho)}=\left(\mathbf{W}_{l, m, p}^{(\rho)}, \mathbf{n}_{m, p}\right), \\
& \hat{\rho}_{l, m} \hat{u}_{l, k, m}=\rho_{l, m} u_{l, k, m}+\frac{\Delta t}{\left|V_{m}\right|} \sum_{p=1}^{M_{m}} W_{l, n, m, p, k}^{(\rho u)}\left|S_{m, p}\right|, \\
& W_{l, n, m, p, k}^{(\rho u)}=\left(\mathbf{W}_{l, m, p}^{\left(\rho u_{k}\right)}, \mathbf{n}_{m, p}\right), \quad k=x, y, z, \\
& \hat{E}_{l, m}=E_{l, m}+\frac{\Delta t}{\left|V_{m}\right|} \sum_{p=1}^{M_{m}} W_{l, n, m, p}^{(E)}\left|S_{m, p}\right|, \\
& W_{l, n, m, p}^{(E)}=\left(\mathbf{W}_{l, m, p}^{(E)}, \mathbf{n}_{m, p}\right), \quad m=1, \ldots, M, \quad l=a, b .
\end{aligned}
$$

Here $f_{m}, \hat{f}_{m}$ - values of the corresponding functions on the lower and upper layers on time in the center of the control volume $V_{m}$ (coinciding into effect of the selected approximation with grid cell $\left.C_{m}\right),\left|V_{m}\right|$ - value of the control volume $V_{m},\left|S_{m, p}\right|$ - faces squares $S_{m, p}$ of the control volume $V_{m}, M_{m}$ - faces number of the control volume $V_{m}, W_{n, m, p}^{(\alpha)}$ - scalar multiplication of flux $\mathbf{W}_{m, p}^{(\alpha)}$ via the face $S_{m, p}$ by the external normal $\mathbf{n}_{m, p}$ to this face $\left(\alpha=\rho, \rho u_{x}, \rho u_{y}, \rho u_{z}, E\right)$. The fluxes approximations $\mathbf{W}_{m, p}^{(\alpha)}$ are similar to the proposals in [10], but do not use artificial viscosity.

\section{Parallel implementation}

Parallel realization uses supercomputers (or clusters) with hybrid architecture $[11,12]$. Such computer systems have several multi-core central processors (CPUs) on each node, as well as several vector or graphics processors (VPUs or GPUs). Parallelization is performed on principles of geometric parallelism, splitting of computational area into subdomains and uses algorithms for dynamic load balancing of the calculators.

The calculation is performed on the discrete QGD equations on a grid distributed among cluster nodes using the "domain decomposition" technique. For a Cartesian grid, when parallelizing, a grid of calculators corresponding to the spatial dimension of the problem is used. For irregular hybrid grid, for parallelization, software packages are applied, such as: METIS [13], ParMETIS [14, 15]. Within each compute node, the grid cells are distributed among the CPU threads by the same algorithms as among the nodes. The QGD computations are strongly coupled and therefore they are executed on the CPU [16].

For computing two systems were implemented:

1) MVS-10P (JSCC RAS). The calculations were performed on the central processors Intel Xeon E5-2690 E5-2690v0@2.90GHz and vector processors Intel Xeon Phi 7110X. The number of CPU cores ranged from 16 to 2048. The number of VPU cores ranged from 60 to 7680. Interconnect Infiniband FDR ( $56 \mathrm{~Gb} / \mathrm{s})$;

2) MVS-5P (JSCC RAS): This system based on Intel Xeon E5-2697A v4@2.60GHz (88 nodes, 2 processors per node, 16 cores per processor, 2 threads per core). Interconnect is OmniPath $(100 \mathrm{~Gb} / \mathrm{s})$.

\section{Results}

An analysis of the numerical results was carried out. The problem was researched in details for $(z, r)$ geometry and $D_{0}=310 \mu \mathrm{m}$. The grids with the number of square $\left(23424 * 2^{\mathrm{k}}\right) \times\left(192 * 2^{\mathrm{k}}\right)$ cells were used (parameter $\mathrm{k}=1$, $2,3)$. The analysis included three questions: accuracy of mathematical model, accuracy of numerical method, efficiency of parallel implementation. 
Firstly, we have compared the QGD and the NavierStokes models. For this purpose, we have calculated the norms of the density difference, pressure difference, temperature difference and velocity difference for some fixed time moments. One result of these calculations is presented in Table 1. Analysis of data showed that the solutions obtained by these models may differ significantly, especially in the areas of rapid development of the flow. However, if the flows are stationary and the Mach numbers are moderate (for example, $0.1 \leq M a \leq 3$ ) then from a certain instant in time, these solutions practically coincide. The difference is in only time to reach the steady-state.

Table 1. Comparison of the models $(\mathrm{t}=8 \mu \mathrm{s})$.

\begin{tabular}{|c|c|c|c|}
\hline $\begin{array}{c}\text { № } \\
\text { grid }\end{array}$ & $\left\|\rho_{Q G D}-\rho_{N S}\right\|_{C}$ & $\left\|p_{Q G D}-p_{N S}\right\|_{C}$ & $\left\|T_{Q G D}-T_{N S}\right\|_{C}$ \\
\hline 1 & $1.49 \mathrm{e}-04$ & $3.61 \mathrm{e}-05$ & $6.70 \mathrm{e}+01$ \\
\hline 2 & $3.33 \mathrm{e}-04$ & $4.86 \mathrm{e}-05$ & $2.61 \mathrm{e}+01$ \\
\hline 3 & $5.43 \mathrm{e}-04$ & $4.31 \mathrm{e}-05$ & $2.14 \mathrm{e}+01$ \\
\hline
\end{tabular}

At second, we have considered the accuracy of the numerical method for the grid sequence. We have compared the norms of the density difference, pressure difference, temperature difference and velocity difference for the grids with the space steps $h, h / 2, h / 4$. The obtained results are presented in Table 2. These results confirm the grid convergence of the numerical procedure.

Table 2. Comparison of the accuracy.

\begin{tabular}{|c|c|c|c|}
\hline Grids & $\left\|\rho_{h}-\rho_{h / 2}\right\|_{C}$ & $\left\|p_{h}-p_{h / 2}\right\|_{C}$ & $\left\|T_{h}-T_{h / 2}\right\|_{C}$ \\
\hline 1 and 2 & $3.25 \mathrm{e}-4$ & $6.34 \mathrm{e}-5$ & $8.21 \mathrm{e}-3$ \\
\hline 2 and 3 & $9.87 \mathrm{e}-5$ & $1.96 \mathrm{e}-5$ & $2.61 \mathrm{e}-3$ \\
\hline
\end{tabular}

At third, we have analyzed the efficiency of parallel implementation of the presented numerical method. Computing times and efficiency of the parallelization are shown in Figures 2 and 3 for $(z, r)$ geometry and grid with $k=2$. These data demonstrate a possibility of calculations of gasdynamic tasks on big configurations. At the same time, sharp falling of efficiency on small configurations illustrates the known problem of data processing of large volume by one thread of CPU. The use of both the CPUs and the VPUs on the same node allows us to reduce the computing time by 1.5-1.7 times. However, the efficiency degrades rapidly because of the VPUs cores have slow network exchanges.

The plots of some numerical results are displayed in Figure 4 and 5. They show the density distribution and concentration distribution obtained at the time $\mathrm{t}=27.6 \mu \mathrm{s}$. Calculations were performed on the supercomputer system MVS10P. The pressure and the temperature profiles do not have strong gradients at the time, and therefore are not shown. In fact, an isothermal flow is established here.

The calculations were carried out in the threedimensional case in the $(\mathrm{z}, \mathrm{x}, \mathrm{y})$ coordinates The main part of the numerical experiments was carried out on a grid with the cubic cells $\left(23424 * 2^{\mathrm{k}}\right) \times\left(384 * 2^{\mathrm{k}}\right) \times(384$ * $2 \mathrm{k}$ ) (parameter $k=1,2,3)$. For this variant, the efficiency of parallelization was tested. It increased for the maximum configurations of used supercomputers by 1.5 times $[17,18]$. At the same time, we can not use VPUs in full due to their small RAM. In generally it is possible to note that results of $3 \mathrm{D}$ and $2 \mathrm{D}$ calculations coincide for average sizes of the flow parameters, but differ in dynamics of the process.

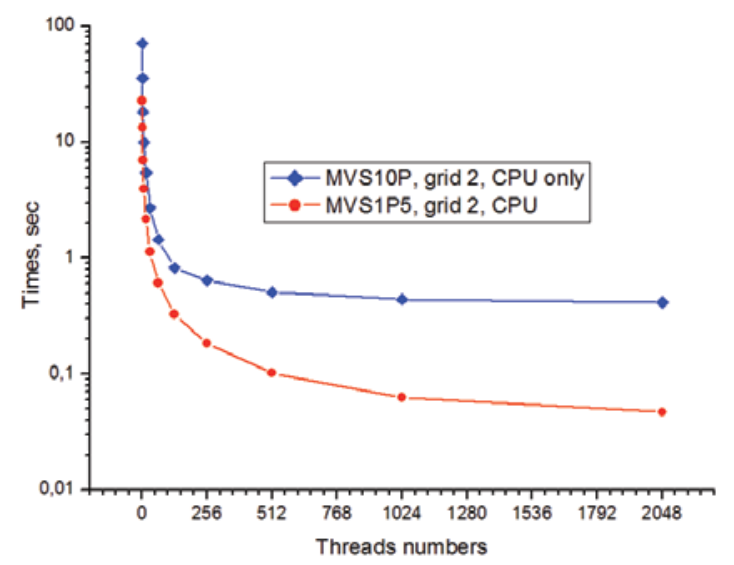

Fig. 2. Computing time.

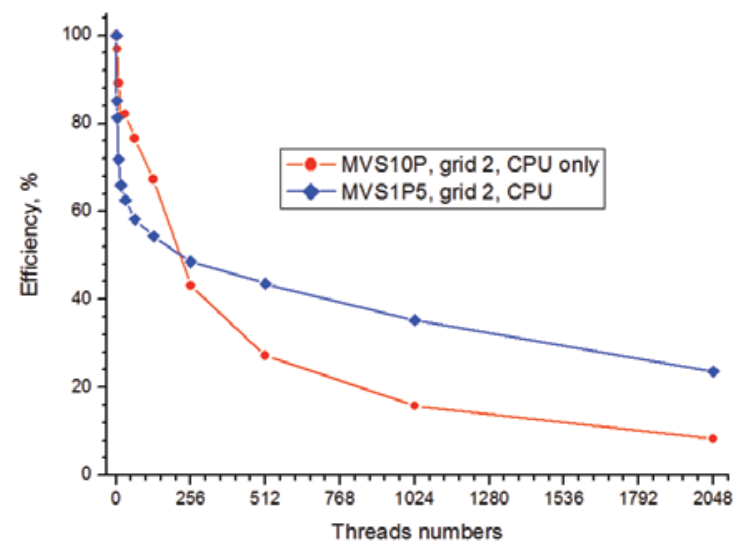

Fig. 3. Efficiency of calculations.

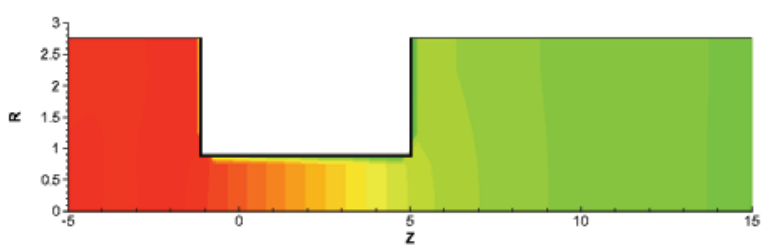

Fig. 4. Gas density distribution near the micro nozzle entry. 


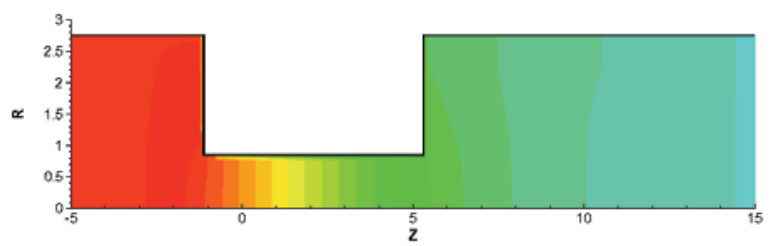

Fig. 5. Decimal logarithm of gas concentration near the micro nozzle entry.

\section{Conclusion}

The problem of gas flows simulation in the micro channels of the technical systems for a real geometry is considered. To solve it, the numerical parallel algorithm is supported. This one is based on the QGD equations and the finite volume method applied to cells of different shapes The algorithm was tested for the case of an axially symmetric and complete three-dimensional Cartesian geometry of the problem. The calculations confirmed the robustness and effectiveness of the developed common approach. The development of the work will be connected with the calculations of the flows for more complex variants. It can be a curvilinear geometry of the computational domain or the use of hybrid grids.

This work was supported by Russian Foundation for Basic Research (grant № 16-07-00206-a, № 17-01-00973-a).

\section{References}

1. D. Resnick. Nanoimprint lithography, in "Nanolithography. The art of fabricating nanoelectronic and nanophotonic devices and systems" / Edited by Martin Feldman. Woodhead Publishing Limited, P. 315-345, (2014).

2. A. Aota, T. Kitamori, Micro- and Nanofluidics, in "Nanofabrication handbook" / Edited by Stefano Cabrini, Satoshi Kawata. CRC Press, 2012. - P. 379398; (2012).

3. T.G. Elizarova Quasi-Gas Dynamic Equations. Springer-Verlag, Berlin Heidelberg New York (2009).

4. B.N. Chetverushkin, Mathematical modeling of problems in the dynamics of a radiating gas, Moscow, Science, In Russian (1985).

5. I.A. Graur, T.G. Elizarova, T.A. Kudryashova, S.V. Polyakov. Numerical investigation of jet flows, using multiprocessor computer systems // J. Mathematical Modelling, 14(6), p. 51-62, (2002).

6. A. Ramos, G. Tejeda, J.M. Fernandez, S. Montero. Nonequilibrium Processes in Supersonic Jets of N2, H2, and N2 + H2 Mixtures: (I) Zone of Silence. J. Phys. Chem. A, 113, p. 8506-8512, (2009).

7. I.V. Fryazinov, Balance method and variationaldifference schemes. Differentsial'nye uravneniya, 7, 1332-1343, (1980).
8. A.A. Samarskiy, A.V. Koldoba, Yu.A. Poveshchenko, V.F. Tishkin, A.P. Favorskiy, Difference schemes on irregular grids. Minsk, Publishing of Closed Joint Stock Company "Criterion", (1996).

9. R. Eymard, T.R. Gallouet, R. Herbin, The finite volume method. Handbook of Numerical Analysis, Amsterdam, North Holland, 7, 713-1020, (2000).

10. I.V. Popov, I.V. Fryazinov, Method of adaptive artificial viscosity for the equations of gas dynamics on triangular and tetrahedral grids. Mathematical Models and Computer Simulations, 1, 50-62, (2013).

11. T.A. Kudryashova, S.V. Polyakov, V. Podryga, Yu. Karamzin. Multiscale modeling of nonlinear processes in technical microsystems. // J. Mathematical modelling, № 7, V 27 (2015).

12. T.A. Kudryashova, S.V. Polyakov, V. Podryga. CFD Problems Solving Parallel Approaches on Supercomputers. 20th International Conference on Circuits, Systems, Communications and Computers (CSCC 2016), Corfu Island, Greece, July 14-17, 2016. N. Mastorakis, V. Mladenov and A. Bulucea (Eds.). MATEC Web of Conferences $\mathbf{7 6}$ 04025(2016).

13. METIS-Family of Multilevel Partitioning Algorithms, http://glaros.dtc.umn.edu/gkhome/ views/metis, (2009).

14. Karypis G., Schloegel K., Kumar V. ParMETIS Parallel Graph Partitioning and Sparse Matrix Ordering Library. URL: http://glaros.dtc.umn.edu/gkhome/metis/parmetis

15. Satish Balay, Kris Buschelman, William D. Gropp, Dinesh Kaushik, Matthew G. Knepley, Lois Curfman McInnes, Barry F. Smith, Hong Zhang, PETSc Web page, http://www.mcs.anl.gov/petsc, (2001).

16. Hang Si, TetGen: A Quality Tetrahedral Mesh Generator, http://tetgen.berlios.de/, (2009).

17. http://www.mcs.anl.gov/research/projects/mpi/

18. http://www.parallel.ru 